\title{
The relation between college students' academic mindsets and their persistence during math problem solving
}

\author{
Chen Shen ${ }^{\mathrm{a}}$, David B. Miele ${ }^{\mathrm{a}}$, Marina Vasilyeva ${ }^{\mathrm{a}, \mathrm{b} *}$ \\ a Lynch School of Education, Boston College, Boston, USA \\ ${ }^{\mathrm{b}}$ Faculty of Psychology, Lomonosov Moscow State University, Moscow, Russia \\ *Corresponding author. E-mail: marina.vasilyeva.1@bc.edu
}

\begin{abstract}
The present study examined the extent to which college students' academic mindsets predicted their persistence when solving challenging math problems. The study included an experimental manipulation, in which participants initially received either an easy or a difficult arithmetic task. Following the manipulation, all participants solved two target math problems: one that was solvable but very hard and another that was unsolvable. Time spent attempting to solve each problem served as a measure of persistence. Results showed the predicted pattern for the solvable target problem, but not for the unsolvable problem. That is, for the solvable problem, the more of a fixed mindset participants had, the less persistent they were after completing a relatively difficult arithmetic task than after completing an easy task. The results suggest that, for certain types of math problems, students' persistence may vary as a function of academic mindset and previous experiences of math success or failure.
\end{abstract}

Keywords: math problem solving, academic mindset, math persistence, math confidence, college students

\section{Introduction}

\section{The relation between college students' academic mindsets and their persistence during math problem solving}

In modern society, where progress largely depends on advances in science and technology, achieving high levels of math understanding and problem-solving skills provides a foundation for both educational and professional success. Conversely, low levels of math achievement in college have been shown to impose constraints on students' career options (e.g., Shapka, Domene, \& Keating, 2006). Thus, in order to optimize educational and professional outcomes, it is critical that we gain a better understanding of why some students perform worse than others in this fundamental area of knowledge.

In order to explain the origin of individual differences in math problem solving, researchers have carried out investigations from multiple perspectives. Some stud- 
ies have focused on variability in cognitive capacity, identifying working memory, attention skills, and other executive functions as correlates of math performance (e.g., Mazzocco \& Kover, 2007; McClelland, Cameron, Connor, Farris, Jewkes, \& Morrison, 2007; Swanson \& Beebe-Frankenberger, 2004). Other studies have examined variability in math strategy instruction, providing evidence that the use of advanced strategies is associated with greater accuracy in math problem solving (e.g., Crosnoe et al., 2010; Vasilyeva, Laski, \& Shen, 2015). However, even when controlling for such cognitive factors, differences in math performance still emerge (e.g., Heaven \& Ciarrochi, 2012). This has led researchers to search for emotional and motivational factors that can potentially explain why students with similar levels of working memory and knowledge of advanced strategies sometimes exhibit different levels of math performance (Covington, 2000; Meece, Anderman, \& Anderman, 2006; Steinmayr \& Spinath, 2009).

\section{Differences in motivation and persistence}

Existing studies have documented a relation between various components of motivation and students' math achievement across levels of education. For example, researchers examined a nationally-representative U.S. sample of middle-school students and found that achievement motivation and attitudes towards math were both directly and indirectly related to math grades (Singh, Granville, \& Dika, 2002). Other scholars examined high-school students and reported that, controlling for intelligence and prior grades, students' attitudes towards math, achievement motives, and goal orientations all predicted subsequent math performance (Steinmayr \& Spinath, 2009). Hackett (1985) tested college students and found that certain aspects of motivation, such as self-efficacy, predicted students' achievement in math courses, as well as their choice of a math-related major. However, it should be noted that most of these studies examined the effect of motivation on broad measures of math achievement, such as grades and standardized test scores. Relatively few studies have directly investigated the effect of motivation on specific math problem-solving behaviors that may account for broad differences in achievement.

One particular problem-solving behavior that is likely to be influenced by motivational factors is persistence, or students' willingness to stay on task even if it requires substantial time and effort to complete. Studies have shown striking differences among students on this characteristic, especially when it comes to math content (e.g., Fredricks, Blumenfeld, \& Paris, 2004; Miller, Greene, Montalvo, Ravindran, \& Nichols 1996). When solving a challenging math problem, some students do not stop trying until they find the solution, whereas others decide to move on to the next task after one or two attempts. Differences in persistence patterns during problem solving may help to explain why students with similar levels of intelligence and instructional input may end up with different educational outcomes (Hardre \& Reeve, 2003). But, what explains persistence? Why do some students persist longer on challenging math problems than others? In the present study, we attempt to address this question by examining the relation between students' "academic mindsets" (their beliefs about the nature of intelligence) and their persistence. 


\section{Predicting persistence: The role of academic mindset}

The concept of academic mindset refers to people's beliefs about whether intelligence can be changed (Dweck, 1999). According to Dweck, there are two basic mindsets regarding intelligence: People with a "fixed" mindset view intelligence as a personal trait that cannot be changed, whereas people with a "growth" mindset view intelligence as a malleable characteristic that can be improved with effort. To be clear, even though the terms "growth" and "fixed" mindsets suggest a dichotomous construct, they are typically measured on a continuum, such that individuals can hold relatively stronger or weaker versions of each mindset. For simplicity of presentation, we will use these terms to refer to individuals who are closer to one end of the continuum than the other.

Individuals who hold these different views tend to behave in different ways when presented with challenging tasks (Hong, Chiu, Dweck, Lin, \& Wan, 1999). In particular, when people with a fixed mindset experience difficulty completing an academic task or receive negative feedback about their performance, they interpret this experience as an indication that they lack the intelligence or ability needed to be successful. And, because they believe that this low level of intelligence is fixed, they are likely to conclude that continuing to work hard will only lead to further embarrassment or will be a waste of their time. In contrast, those who hold a growth mindset tend to interpret the experience of difficulty or negative feedback as a sign that they may need to work even harder. The more time and effort they spend, the more their intelligence or ability will improve. Consequently, people with a growth mindset are more likely than people with a fixed mindset to persist at challenging tasks.

Several studies have investigated this proposed relation between mindsets and persistence. For instance, Hong and colleagues (Hong et al., 1999; Study 3) used fake scientific articles to temporarily shift participants' beliefs toward either the fixed or the growth end of the mindset continuum and then had participants complete problems from an intelligence test. After the test, half of the participants were told that they had performed well $\left(66^{\text {th }}\right.$ percentile), while the other half were told that they had performed poorly $\left(20^{\text {th }}\right.$ percentile). Participants were then asked whether they would like to engage in a tutorial exercise that had been shown to improve performance on the test or in an unrelated ability task. Choosing to work on the tutorial exercise could be construed as a sign of task persistence. The results showed that most participants who were shifted toward a growth mindset chose to engage in the tutorial exercise (73.3\%), regardless of what kind of performance feedback they received. In contrast, participants who were shifted toward a fixed mindset were much less likely to engage in the tutorial task after receiving negative feedback (13.3\%) than after receiving positive feedback (66.7\%).

Another study (Liu, Chiu, Chen, \& Lin, 2014) employed a correlational design, in which high-school students completed questionnaires measuring their academic mindsets, as well as their general willingness to confront challenges. The results showed that participants with a growth mindset reported stronger challenge-confronting tendencies. In addition, O'Shea and colleagues (O'Shea, Cleary, \& Breen, 2010) conducted a study exploring the relation between goal-orientation, self-confidence, academic mindsets and persistence on math tasks. They measured aca- 
demic mindsets using a mix of math-specific and more general items and measured persistence in terms of participants' self-reported preference for challenging math tasks, as well as their willingness to work hard on such tasks. The results showed that the more the students endorsed a growth mindset, the more they reported persisting on challenging math tasks.

In addition to measuring self-reported task preferences and levels of effort, studies of academic mindsets have also measured persistence in terms of students' actual behavior. Heine and colleagues (2001) conducted a cross-cultural study of mindsets that used a similar paradigm as Hong et al. (1999), except that (a) they measured college students' more general mindsets regarding personality as opposed to more specific mindsets regarding intelligence (e.g., "Everyone is a certain kind of person and there is not much that can be done to really change that") and (b) they measured persistence in terms of how much time participants spent completing a similar task after receiving success or failure feedback on the original task. The results showed that, for both American and Japanese participants, there was no association between mindsets and persistence after success feedback, but that the more the students endorsed a growth mindset, the more they persisted after receiving failure feedback.

Finally, in a study by Cury and colleagues (Cury, Fonseca, Zahn, \& Elliot, 2008), younger participants (age 13-15) received success or failure feedback after completing items from an IQ test. They were then experimentally shifted toward a growth or fixed mindset regarding their ability being measured by the test. Before being given a second opportunity to complete the test, they were given an opportunity to practice for five minutes. Persistence was measured in terms of how much of the five minutes the participants spent practicing. The results showed that participants in the fixed mindset condition spent less time practicing and performed worse on the subsequent test than participants in the growth mindset condition.

In sum, there are several studies that examined the relation between academic mindsets and persistence in non-math domains using behavioral measures of persistence, and at least one study that investigated this relation in the math domain, although using self-report measures. To the best of our knowledge, there have been no published studies examining the relation between academic mindsets and actual behavioral persistence within the domain of math problem solving. This is the focus of the present study.

\section{The present study}

In this study, we examined the extent to which college students' academic mindsets predicted their persistence when solving challenging math problems. As noted earlier, academic mindsets should be particularly predictive of persistence when individuals are responding to challenge or negative feedback. In order to induce different levels of challenge, we experimentally manipulated the difficulty of an initial set of math problems that the students completed prior to being presented with the target problems. In one condition (Low Challenge), participants had to solve simple addition problems, and in the other condition (High Challenge), they had to solve more difficult multiplication problems in a limited amount of time. 
After completing the challenge manipulation, participants were asked to solve two target problems. Some researchers may attempt to measure persistence in terms of the total time students spend completing a large number of problems that can be solved without too much difficulty. However, this way of measuring persistence is problematic because more competent individuals may find solutions more quickly than less competent individuals, and thus appear to be less persistent even though they are just as motivated to successfully complete the task. A partial solution to this problem involves presenting participants with extremely challenging (and perhaps even unsolvable) problems that require substantial time and effort even from highly competent individuals. Thus, we conducted pilot work to identify two problems that satisfied this criterion. Both problems utilized mathematical concepts that are typically acquired in middle school. Yet, their difficulty was high: One of them could not be solved in principle and the other was a solvable problem, which required multiple steps, as well as insight, to arrive at a solution. In fact, none of our pilot subjects (who were drawn from the same pool as study participants) could solve this problem.

Based on previous findings from the academic mindset literature (reviewed above), we expected students with a fixed mindset to spend less time solving the target math problems (i.e., not to persist as long) after completing a challenging math task (High Challenge condition) than after completing a relatively easy math task (Low Challenge condition). In contrast, we did not expect participants with a growth mindset to persist less in the High Challenge condition; if anything, we expected them to persist longer after completing a challenging task.

\section{Method}

The current study was part of a larger project that collected participants' data on multiple measures. Here we focus only on those measures that were relevant to our research question and on the participants who completed all these measures.

\section{Participants}

A total of 188 participants completed the first part of the study. Of these, 48 either did not complete the second part or completed a previous version of the second part with different target problems. Two additional participants did not complete the primary independent measure (of academic mindsets), and one participant was missing data due to computer error. The final sample included 137 college students, majoring in education or psychology, from a private research university in Massachusetts. The students were given a course credit for their participation. Among the 137 participants, $83 \%$ were female students, reflecting the gender composition of their respective departments. The age of participants varied between 18 to 24 years, with a mean age of 19 . The majority of the participants were European-American (71\%) and the rest were African-American, Asian-American, Hispanic-American or multiracial. 


\section{Materials}

Theory of intelligence questionnaire. We used Dweck's Implicit Theory of Intelligence Questionnaire (Dweck, 1999) to measure academic mindsets. It included eight items describing intelligence as being fixed (e.g., "You can learn new things, but you can't really change your basic intelligence") or malleable (e.g., "You can always substantially change how intelligent you are"). Participants read each statement and indicated their level of agreement on a 6-point scale $(1=$ "strongly disagree" to $6=$ "strongly agree"). To arrive at a score for each participant, we first reverse-coded the items reflecting a growth-oriented view of intelligence so that higher scores reflected a greater tendency towards a fixed mindset. Then we averaged the participant's ratings across the eight items. To prepare the scores for statistical analysis, we standardized them relative to the midpoint (by subtracting the midpoint value of 3.5 from a given score and dividing the result by the standard deviation of the sample). Thus, we created an index of the participants' academic mindsets, with positive values suggesting a fixed mindset and negative value suggesting a growth mindset. The reliability of the mindset scale in our study was high (Cronbach's $\alpha=.93$ ).

Challenge manipulation task. We created two sets of arithmetic problems that varied in difficulty so as to induce divergent effort experiences. Materials for the Low Challenge condition included three double-digit addition problems (e.g., $58+34$ ), while materials for the High Challenge condition included six double-digit multiplication problems (e.g., $18 \times 16)$. Participants were instructed to complete these problems mentally (i.e., without paper, pencil, or calculator) and were given 4 minutes to do so. The relative difficulty of each condition, which was initially based on logical considerations, was supported by our pilot data.

Challenge manipulation check. After completing the math persistence task, participants answered two questions about the amount of effort and the difficulty level they experienced when solving the mental arithmetic problems during the effort manipulation task, as well as two questions about the amount of effort and the difficulty level they experienced during the math persistence task: "How much effort did you put into solving the initial set of arithmetic problems [the second set of math problems]?" ( $1=$ "no effort" to $7=$ "a lot of effort") and "How difficult was it for you to solve the initial set of arithmetic problems [the second set of math problems]?" ( $1=$ "not at all difficult" to $7=$ "very difficult"). Even though we had pilot data differentiating the two experimental conditions, we used this measure to check whether our participants indeed had a different effort experience and whether these differences were consistent with our expectations.

Math confidence measure. After completing the challenge manipulation, and again after completing the math persistence task, participants answered two questions about their math ability: "How good are you at solving math problems?" ( $1=$ "not at all good" to $7=$ "very good") and "How confident are you in your math ability?" ( $1=$ "not at all confident" to $7=$ "very confident"). The initial administration of this measure was meant to examine the extent to which participants called into question their math ability after completing the multiplication problems in the High Challenge condition, compared to the easier addition problems in the Low Challenge condition. Prior research (see Muenks \& Miele, 2016) suggests that 
individuals with a fixed mindset are more likely than individuals with a growth mindset to perceive a negative relation between effort and ability. Thus, it was possible that participants with a fixed mindset would report less confidence in their math ability in the High Challenge condition than in the Low Challenge condition, whereas participants with a growth mindset would be equally confident across the two conditions, or perhaps more confident in the High Challenge condition (if they perceived their hard work as improving their math ability). On the other hand, if participants' experience of the High Challenge condition was marked by failure, it is possible that all participants (regardless of mindset) would call their math ability into question and express low confidence.

Math persistence task. This task consisted of two math problems (see Appendix), which were selected through intensive piloting, and were found to be challenging. As noted earlier, one of the problems had a solution and the other one was unsolvable. We included an unsolvable problem so that participants could spend as much time as they wanted to devote to that problem without finding a solution. The amount of time participants spent on each problem was used as the measure of persistence.

Demographic questionnaire. A brief questionnaire was used to collect information about demographic characteristics of our sample, including gender, age, and ethnic background.

\section{Procedure}

Participants took part in two testing sessions, which were conducted an average of 15.7 days apart ( $\mathrm{SD}=3.7$; range: 10 to 33 days). All tasks in both sessions were administered on computers, using Qualtrics Survey Software, by trained research assistants. In Session 1, participants finished a battery of self-report measures, including the theory of intelligence questionnaire. The entire battery took about 15 minutes to finish.

Session 2 included an effort manipulation task and a math persistence task. As part of the effort manipulation task, participants were randomly assigned to either the Low Challenge or High Challenge condition. In both conditions, they were given 4 minutes to solve mental arithmetic problems. If participants finished earlier, they could move on by clicking on the "Continue" button. If they were still working after 4 minutes had passed, the program automatically advanced them to the next screen.

Following the effort manipulation, participants from both conditions answered the first set of math confidence questions. They then took part in the same math persistence task, which involved solving two math problems, presented in a counterbalanced order. They were informed that they could stop and move on to the next problem at any point, but would not be able to click back from the second problem to the first problem. Participants were given 16 minutes to solve each problem, though they were not informed about this time limit. If participants were still working on a problem at the end of the 16-minute period, the program automatically advanced them to the next screen. After attempting to solve each problem, participants were given two minutes to explain their solution. 
When students were done with the math problems, they were presented with the second set of math confidence questions, followed by the challenge manipulation check. Finally, they completed the demographic questionnaire. Session 2 lasted between 15 and 40 minutes, depending on how much time the participant spent on the math persistence task.

\section{Results}

In analyzing the data, we first examined the results of the manipulation check to make sure that our experimental manipulation was effective at inducing different effort experiences in participants across the two conditions. Next, to test our key research hypotheses concerning the relation between academic mindsets and persistence, we conducted a series of ANCOVAs. We conducted ANCOVAs instead of linear regression analyses because some of our analyses included repeated measures; and ANCOVA, unlike regression, allowed us to examine the interaction between a continuous independent variable and a repeated measures factor without having to implement multi-level modeling.

The analyses that included a repeated measures factor (e.g., problem type) along with a continuous predictor (academic mindsets) were conducted in three steps (see Miele, Son, \& Metcalfe, 2013). In Step 1, we conducted a mixed ANOVA with challenge condition as a between-subject factor and problem type as a repeated measure factor. In Step 2, we added the academic mindsets index in order to conduct a mixed ANCOVA. The main effect of problem type was reported from the first step in order to maximize power and to avoid scaling artifacts (see Algina, 1982; Thomas, 2009; Thomas et al., 2009). The main effects of challenge condition and academic mindsets, as well as all two- and three-way interactions, were reported from the second step. In Step 3, we carried out further analyses to explore any significant interactions between challenge condition and academic mindsets index. These simple-effect analyses estimated the effects of the challenge condition on persistence for participants who scored above (1.5 SD) and below (1.5 SD) the midpoint on the academic mindsets index (Aiken \& West, 1991).

For analyses that did not include a repeated measures factor (e.g., analyses of time spent on the challenge manipulation task), we conducted one-step ANCOVAs. Note that because the academic mindsets index included in the ANCOVAs was midpoint centered, all main effects of the challenge condition, as well as all Challenge Condition $\times$ Repeated Measure interactions, were estimated for participants who scored at the midpoint of the academic mindsets index, as opposed to participants who scored at the mean of the academic mindsets index. This scaling of the covariate can affect the significance of the challenge condition effects, particularly if the higher order interaction involving the academic mindsets index happens to be significant.

\section{Manipulation check}

To begin with, we analyzed participants' behavior on the math items in each condition of the challenge manipulation task. Participants in the Low Challenge condition were able to answer all problems in 4 minutes with $93 \%$ accuracy, whereas 
participants in the High Challenge condition answered, on average, $75 \%$ of problems in the same time period, with only $33 \%$ accuracy on the problems answered and 23\% accuracy overall. This suggests that many of the participants in the High Challenge condition did not have enough time to accurately complete all of the math problems they were assigned. To formally examine differences in time spent solving problems, we submitted time to an ANCOVA, with Challenge Condition as a between-subjects factor and Academic Mindset as a continuous covariate predictor $^{1}$. The results of this analysis revealed a main effect of challenge condition: participants spent more time in the High Challenge condition than in the Low Challenge condition, $F(1,133)=285.02, p<.001, \eta_{\mathrm{p}}{ }^{2}=.68$. There was no main effect of Academic Mindset, nor was there a Challenge Condition $\times$ Academic Mindset interaction ( $p$ 's $>.23)$.

In addition to analyzing participants' actual behavior and performance on the math items, we examined their subjective responses to the two questions concerning the amount of effort they expended and the amount of difficulty they experienced. We submitted responses to each question to a 2 (Task Focus: Challenge Task vs. Persistence Task) $\times 2$ (Challenge Condition: Low Challenge vs. High Challenge) mixed ANCOVA, with repeated measures on the first factor and the Academic Mindset index as a covariate. This allowed us to determine whether the challenge manipulation affected participants' perceptions of effort and difficulty on the initial challenge task, as well as whether this effect happened to carry over to participants' perceptions of effort and difficulty on the persistence task.

For the effort question, there were significant main effects of Task Focus, $F(1,135)=35.25, p<.001, \eta_{\mathrm{p}}{ }^{2}=.21$, and Challenge Condition, $F(1,133)=5.56$, $p=.02, \eta_{\mathrm{p}}{ }^{2}=.04$; however, these effects were qualified by a significant Task Focus $\times$ Challenge Condition interaction, $F(1,133)=7.57, p=.007, \eta_{\mathrm{p}}{ }^{2}=.05$. Additional simple effects analyses showed that, for the Challenge Task, participants reported expending significantly more effort in the High Challenge condition $(M=4.76$, $S E=.23)$ than in the Low Challenge condition $(M=3.71, S E=.22), t(133)=3.34$, $p=.001 .{ }^{2}$ However, for the Persistence Task, participants reported expending relatively high levels of effort in both conditions (High Challenge: $M=5.39, S E=.20$; Low Challenge: $M=5.36, S E=.19), t(133)=.04, p=.97$. None of the effects involving academic mindsets as a predictor were significant ( $p$ 's $>.29$ ).

The pattern of results was similar for the difficulty question: There were significant main effects of Task Focus, $F(1,135)=101.04, p<.001, \eta_{\mathrm{p}}{ }^{2}=.43$, and Challenge Condition, $F(1,133)=26.72, p<.001, \eta_{\mathrm{p}}{ }^{2}=.17$; however, these effects were qualified by a significant Task Focus $\times$ Challenge Condition interaction, $F(1,133)=26.58$, $p<.001, \eta_{\mathrm{p}}{ }^{2}=.17$. Additional simple effects analyses showed that, for the Challenge Task, participants reported experiencing significantly more difficulty in the High Challenge condition $(M=5.61, S E=.27)$ than in the Low Challenge condition $(M=3.42, S E=.25), t(133)=5.96, p<.001$. However, for the Persistence Task, par-

1 Because the distribution of times exhibited both floor and ceiling effects (due to the relatively short time limit), it did not benefit from a square root transformation. Therefore, unlike our later analyses of time, we included the raw times in our analysis.

2 We report estimated means and SEs based on the full ANCOVA model, controlling for academic mindsets at the midpoint of the scale. 
ticipants reported experiencing relatively high levels of difficulty in both conditions (High Challenge: $M=6.31, S E=.16$; Low Challenge: $M=6.16, S E=.15$ ), $t(133)=.69$, $p=.49$. None of the effects involving Academic Mindset as a predictor were significant $(p$ 's $>.14)$.

Together, findings from the behavioral and self-report analyses demonstrate that the manipulation task successfully induced divergent experiences of challenge in participants, and showed that this effect did not differ as a function of their academic mindset.

\section{Math confidence}

To examine how the challenge manipulation affected participants' math confidence, we submitted this measure to a 2 (Time Point: After Challenge manipulation vs. After Persistence Task) $\times 2$ (Challenge Condition: Low Challenge vs. High Challenge) mixed ANCOVA, with repeated measures on the first factor and Academic Mindset as a covariate. The results of the analysis revealed significant main effects of Time Point, $F(1,135)=110.20, p<.001, \eta_{\mathrm{p}}{ }^{2}=.45$, and Academic Mindset, $F(1,133)=9.77$, $p=.002, \eta_{\mathrm{p}}{ }^{2}=.07$, as well as a marginal effect of Challenge Condition, $F(1,133)=3.17$, $p=.08, \eta_{\mathrm{p}}{ }^{2}=.02$. The main effects were qualified by a significant Time Point $\times$ Challenge Condition interaction, $F(1,133)=4.38, p=.04, \eta_{\mathrm{p}}{ }^{2}=.03$, and a Challenge Condition $\times$ Academic Mindset interaction, $F(1,133)=5.98, p=.02, \eta_{\mathrm{p}}{ }^{2}=.04$ (but not a Time Point $\times$ Challenge Condition interaction, $p=.38$ ). The two-way interactions were in turn qualified by a significant Time Point $\times$ Challenge Condition $\times$ Academic Mindset interaction, $F(1,133)=5.39, p=.02, \eta_{\mathrm{p}}{ }^{2}=.04$.

The nature of the three-way interaction suggested that the effects of challenge condition and participants' academic mindsets on math persistence varied by time point. To explore this possibility, we conducted separate follow-up analyses for each time point, with Challenge Condition as a between-subject variable and Academic Mindset as a covariate. For the first time point (immediately after the challenge manipulation), there were significant main effects of Challenge Condition, $F(1,133)=6.61, p=.01, \eta_{\mathrm{p}}{ }^{2}=.05$, and Academic Mindset, $F(1,133)=11.34, p<.001$, $\eta_{\mathrm{p}}{ }^{2}=.08$, which were qualified by a significant Challenge Condition $\times$ Academic Mindset interaction, $F(1,133)=10.91, p=.001, \eta_{\mathrm{p}}{ }^{2}=.08$. However, for the second time point (immediately after the persistence task), there was a main effect of Academic Mindset, $F(1,133)=5.87, p=.02, \eta_{\mathrm{p}}{ }^{2}=.04$, but not effects of Challenge Condition and no Challenge Condition $\times$ Academic Mindset interaction ( $p$ 's $>.20)$.

To explore the significant interaction for the first time point, we conducted additional simple slope analyses. As shown in Figure 1, one analysis estimated that participants with a growth mindset (1.5 SD below the midpoint of the academic mindsets scale) in the High Challenge condition were as confident as participants with a growth mindset in the Low Challenge condition, $t(133)=1.55, p=.12$. In contrast, participants with a fixed mindset (1.5 SD above the midpoint of the academic mindsets scale) in the High Challenge condition were significantly less confident than participants with a fixed mindset in the Low Challenge condition, $t(133)=3.50, p<.001$. Thus, it appears that the high levels of effort and difficulty briefly experienced by participants in the High Challenge condition during the challenge manipulation task led those with a fixed mindset, but not those with a 

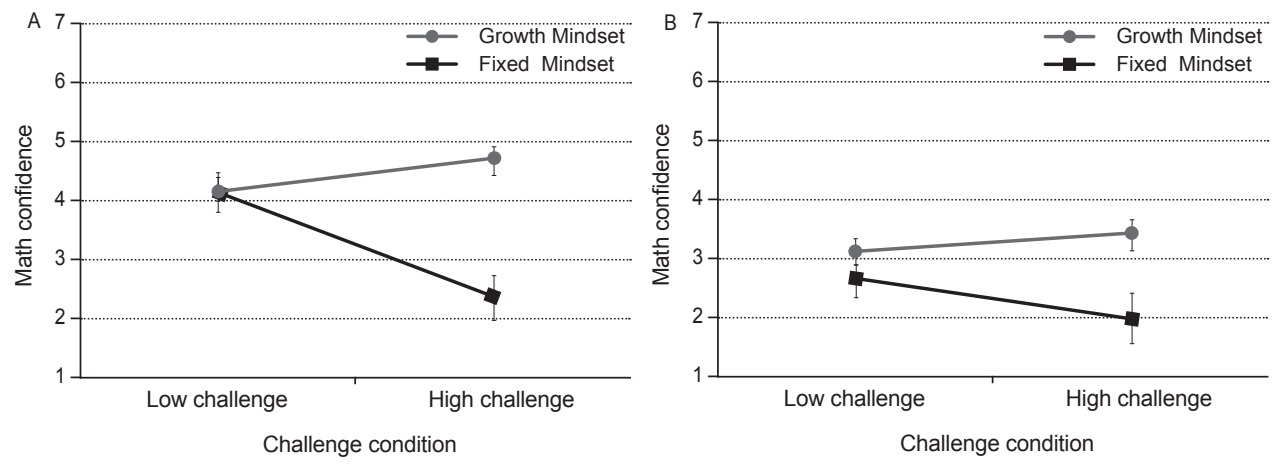

Figure 1. Math confidence by Challenge Condition and Academic Mindset, immediately after the challenge manipulation (A) and after the persistence task (B). Error bars indicate one standard error

growth mindset, to call their math ability into question. Interestingly, most participants (including those with a growth mindset) appeared to call their math ability into question after the longer and more challenging math persistence task, regardless of which version of the challenge task the student initially completed.

\section{Persistence}

There was wide variability (from 20 seconds to 16 minutes) in the amount of time participants spent solving each of the two target problems. On average, they spent about 332 seconds $(S D=266.00)$ working on the solvable problem and 437 seconds $(S D=290.46)$ working on the unsolvable one. This variable was positively skewed: For both problems, most participants spent less than 400 seconds (73\% for the solvable problem, and $54 \%$ for the unsolvable problem), but a few participants used the maximum amount of time that was allowed ( $8 \%$ for the solvable problem and $10 \%$ for the unsolvable problem). To address the issue of skewness, we carried out square-root transformations. For all subsequent analyses, we used the square-root transformed time as a persistence measure for both problems.

An analysis of problem-solving time yielded a significant main effect of Problem Type, $F(1,135)=19.52, p<.001, \eta_{\mathrm{p}}{ }^{2}=.13$, such that participants spent more time on the unsolvable problem than the solvable one. This main effect was qualified by a marginally significant Challenge Condition $\times$ Problem Type interaction, $F(1,133)=2.84, p=.09, \eta_{\mathrm{p}}^{2}=.02$, which was itself qualified by a marginally significant Challenge Condition $\times$ Problem Type $\times$ Academic Mindset interaction, $F(1,133)=3.55, p=.06, \eta_{\mathrm{p}}{ }^{2}=.03$. No other effects were significant $\left(p^{\prime} s>.23\right)$.

The nature of the three-way interaction suggested that the effects of the challenge condition and participants' academic mindsets on math persistence varied for the solvable and unsolvable problem. To explore this possibility, we conducted separate follow-up analyses for each problem, with challenge condition as a between-subject variable and academic mindsets as a covariate. For the unsolvable problem, we found no main effects of Challenge Condition or Academic Mindset (all $p$ 's $>.51)$, nor a significant interaction $(p=.997)$. The pattern of results for the solvable problem, however, was quite different. In particular, although neither 


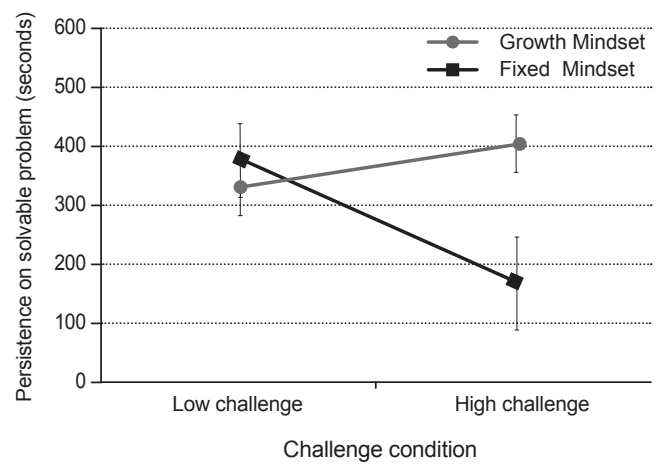

Figure 2. Persistence on solvable problem (in seconds) by Challenge Condition and Academic Mindsets. To make interpretation more straightforward, the figure depicts means that were estimated from a model that included untransformed persistence times, not the model reported in the results section. Error bars indicate one standard error

of the main effects were significant ( $p$ 's $>.14$ ), there was a significant Challenge Condition $\times$ Academic Mindset interaction, $F(1,133)=4.00, p<.05, \eta_{\mathrm{p}}{ }^{2}=.03 .{ }^{1}$

As shown in Figure 2, an additional simple-effects analysis estimated that participants with a growth mindset (1.5 SD below the midpoint of the mindset scale) spent roughly equal amounts of time solving the math problem across the two challenge conditions, $t(133)=1.06, p=.29$. In contrast, another analysis estimated that participants with a fixed mindset (1.5 SD above the midpoint of the mindset scale) spent significantly less time solving the math problem in High Challenge condition compared to the Low Challenge condition, $t(133)=2.04, p=.04 .^{2}$

It should be noted that, after conducting the analyses reported above, we conducted an additional set of analyses that included Problem Order (Solvable First vs.

1 When initially analyzing the data, we applied a more conservative filter which excluded 7 participants who expressed suspicion that one or more of the problems was unsolvable, 4 participants who were particularly slow on the Low Challenge version of the manipulation task, and 2 participants who were particularly fast on the High Challenge version. We also excluded 1 participant who seemed to mistakenly think that the time allowed on the persistence task was only 4 minutes, 1 participant who expressed concern about being sick, and 1 participant who expressed concern about having a learning disability ( $N=16$ in total). In the analysis of persistence, the three-way interaction was marginally significant $(p=.06)$, but the Challenge Condition $\times$ Academic Mindset interaction for persistence on the solvable question was not significant $(p=.12)$ and the effect size was smaller than in the analysis reported above $\left(\eta_{\mathrm{p}}{ }^{2}=.02\right)$.

2 In addition to measuring participants' mindsets about intelligence using the standard Dweck measure, we also measured participants' specific beliefs about math ability using a 4-item measure we adapted from Kloosterman \& Stage (1992). With this measure we found a marginal interaction of problem type and math beliefs on persistence, $F(1,133)=2.90, p=.09, \eta_{\mathrm{p}}{ }^{2}=.02$, but no effect of challenge condition. The interaction reflected the fact that there was a significant inverse correlation between math beliefs and persistence on the solvable problem $(r=-.18, p=.04)$, but not the unsolvable problem $(r=-.02, p=.81)$. It is unclear why the measure of math-specific beliefs (unlike the more general mindset measure) did not interact with the effort manipulation. Perhaps, because the math-specific measure contained items that focused more on the relation between effort and math ability, as opposed to the malleability or fixedness of ability, participants who were high on this measure (i.e., who believed that math ability could not be improved through effort) did not necessarily believe that high effort meant that they were low in ability. 
Unsolvable First) as a factor. Although Problem Order did not significantly moderate the marginally significant three-way interaction $(p=.11)$, it did make the threeway interaction non-significant $(p=.17)$ when included. There was also a marginally significant Problem Order $\times$ Problem Type interaction $(\mathrm{p}=.099)$, as well as a significant Problem Order $\times$ Challenge Condition interaction $(\mathrm{p}=.009)$. To further explore these order effects, we conducted another analysis of the solvable problem, with Problem Order included. Although there was a marginally significant Problem Order $\times$ Challenge Condition interaction $(p=.07)$, the Challenge Condition $\times$ Academic Mindset interaction only dropped slightly in significance $(p=.06)$. Furthermore, this two-way interaction was not moderated by Problem Order $(p=.75)$. In contrast, a final analysis of the unsolvable problem did reveal a marginally significant Problem Order $\times$ Challenge Condition $\times$ Academic Mindset interaction $(p=.06)$, which qualified a significant Problem Order $\times$ Challenge Condition interaction $(p=.008)$. Because we believe that there may have been issues with how participants interpreted the unsolvable problem (e.g., realizing that it was indeed unsolvable; see Discussion), we refrain from interpreting these interactions.

\section{Mediation analysis}

To determine whether the interactive effect of Academic Mindset and Challenge Condition on persistence (for the solvable problem) was partly mediated by participants' math confidence after completing the challenge manipulation, we conducted a mediation analysis using the PROCESS bootstrapping procedure developed by Hayes (2013) and implemented as a macro in SPSS. We used Model \#8 with 5,000 bootstrap resamples and specified the square-root transformed time for the solvable problem as the outcome variable (Y), Academic Mindset as the independent variable (X), Challenge Condition as the moderator (W), and Math Confidence after the challenge manipulation as the mediator $(\mathrm{M})$. The confidence intervals $(-1.71,-.19)$ for the mean indirect effect of the Academic Mindset $\times$ Challenge Condition interaction on persistence through Math Confidence (-.75) did not include 0 , indicating that the effect was significant at $p<.05$ and providing evidence of mediation.

\section{Discussion}

The key research question of the present study was whether students with different mindsets regarding the nature of intelligence tend to show different patterns of persistence when solving math problems. To activate the motivational consequences of holding a particular mindset, we presented one groups of participants with a set of difficult arithmetic problems that were meant to induce high levels of effort and difficulty. Their interpretation of this experience was expected to undermine persistence in students with a fixed mindset, but not in participants with a growth mindset. The remaining participants were presented with simple arithmetic problems, which were not meant to induce high levels of effort or difficulty, and thus were not expected to undermine the persistence of participants with a fixed mindset. Our findings provide support for the hypothesized relation, indicating that the more fixed the participants' mindset, the less persistence they exhibited in the High 
Challenge condition compared to the Low Challenge condition. It is important to note, however, that this effect was qualified by the type of problem, such that the hypothesized relation was only observed in the case of a solvable math problem. It is also worth noting that the effect appears to have been partly mediated by changes in participants' math confidence. That is, participants with a fixed mindset were estimated to have substantially lower confidence in their math ability after completing the High Challenge version of the arithmetic than after completing the Low Challenge version; this lower confidence appears to have translated into less time spent on the solvable problem of the persistence task. Overall, our findings are consistent with previous research, and provide new evidence from the domain of math problem solving, suggesting that students' academic mindsets influence their interpretation of their math problem-solving experiences as well as their persistence on subsequent problem solving.

Although this is not an experimental study, it raises a possibility that shifting one's mindset may have beneficial effects on persistence in a math problem-solving context. Researchers have successfully manipulated participants' mindsets both temporarily and longitudinally and observed divergent patterns on behavior after this manipulation. For instance, some researchers have manipulated participants' mindsets temporarily by presenting them with articles arguing for either a fixed or growth mindset and shown that these mindsets can have a causal effect on participants' subsequent behavior or judgments across multiple domains (e.g., Aronson, Fried, \& Good, 2002; Chiu, Hong, \& Dweck, 1997; Hong et al., 1999; Levy, Stroessner, \& Dweck, 1998; McConnell, 2001). In addition, mindset has been manipulated longitudinally. Blackwell and colleagues (Blackwell, Trzesniewski, \& Dweck, 2007) developed an intervention for middle-school students and followed them over the course of a semester. Their results showed that those who received the mindset intervention not only expressed more of a growth mindset, but also exhibited higher achievement in math (see also Paunesku et al., 2015). These studies suggest that students' mindsets are amenable to intervention in classroom settings.

The malleability of academic mindsets points to an additional path for improving students' persistence, which both teachers and researchers identify as a critical aspect of student behavior during math problem solving. Thus, the current findings linking mindset and persistence in math problem solving have potentially important practical implications. In addition, they have theoretical implications: It is critical to create a comprehensive model of individual differences in math performance, and our findings add information about an important motivational component of this future model.

\section{Difference in the patterns observed with a solvable versus unsolvable problem}

We included an unsolvable problem in the current study so that students with high math skills would be given an opportunity to demonstrate the extent of their persistence without any limits imposed by their own ability to solve the problem quickly. Yet, there was also a possibility that particularly insightful students could see, after some initial attempts, that the problem could not be solved in principle, and thus quit trying for a reason other than a lack of persistence. Thus, we also in- 
cluded a second - solvable - problem that was very challenging and unlikely to be solved in 16 minutes by most participants.

The study participants showed different patterns of performance on the two problems. First, they spent on average more time on the unsolvable problem, compared to the solvable one. This could partly be due to the difference in the nature of the problems. The solvable problem can be categorized as requiring an insight (see Appendix). It might have appeared to participants that some provisions were missing, making them feel that they were stuck in the middle of the problem-solving process, without a clue to figure their way out. Thus, they might have been particularly disposed to quit solving the problem. The unsolvable problem, on the other hand, was likely to elicit a trial-and-error approach, making participants feel that every time they crossed out an unsuitable answer, they were getting closer to the real solution. This sense of making progress might have helped participants stay engaged in the task for a longer time.

The second difference in students' performance on the two problems concerned the hypothesized interaction between the mindset and the challenge condition. Whereas the persistence times on the solvable problem revealed such an interaction, it was not observed in the case of the unsolvable problem. This finding was somewhat puzzling. One possibility is that some participants, including those with a growth mindset, after making a number of attempts to solve the problem, realized that it was unsolvable. Indeed, several study participants made an explicit comment that the problem had no solution (though some participants also said this about the solvable problem). After identifying the problem as unsolvable, these participants may have quit, but not because of a low level of persistence. Thus, the actual level of persistence, measured by the time spent on this problem, may have been confounded with the ability to recognize the problem as unsolvable. This, of course, is just one possible explanation of the lack of effects of mindset and challenge condition on the unsolvable problem. In future work, it would be useful to systematically vary this and other characteristics of the problem in order to determine whether some features of math problems moderate the relation between mindset and persistence on math problems.

\section{Limitations and future directions}

One limitation of this study is that the relation between on-task persistence and math performance was not tested. A more comprehensive study is needed to explore the relation among academic mindsets, on-task persistence, and math problem-solving accuracy. For instance, on-task persistence may serve as a mediator of the effects of academic mindsets on math problem-solving accuracy, as well as long-term math achievement, thus explaining one potential mechanism for the effects observed in previous studies (e.g., Blackwell et al., 2007). Another limitation is that participants' academic mindsets were not manipulated, but measured as an individual difference variable. To draw a causal conclusion regarding the relation between academic mindsets and persistence, an experimental design that manipulates participants' mindsets is necessary. In particular, future work may include a design of an intervention study that targets students' mindsets and tracks longitudinally the effects of the intervention on students' persistence across multiple math tasks. 


\section{References}

Aiken, L. S., \& West, S. G. (1991). Multiple regression: Testing and interpreting interactions. Newbury Park, CA: Sage.

Algina, J. (1982). Remarks on the analysis of covariance in repeated measures designs. Multivariate Behavioral Research, 17, 117-130. doi: 10.1207/s15327906mbr1701_8

Aronson, J., Fried, C. B., \& Good, C. (2002). Reducing the effects of stereotype threat on African American college students by shaping theories of intelligence. Journal of Experimental Social Psychology, 38(2), 113-125. doi: 0.1006/jesp.2001.1491

Ashcraft, M. H. (2002). Math anxiety: Personal, educational, and cognitive consequences. Current Directions in Psychological Science, 11(5), 181-185.doi: 10.1111/1467-8721.00196

Blackwell, L. S., Trzesniewski, K. H., \& Dweck, C. S. (2007). Implicit theories of intelligence predict achievement across an adolescent transition: A longitudinal study and an intervention. Child Development, 78(1), 246-263. doi: 10.1111/j.1467-8624.2007.00995.x

Borman, G. D., \& Overman, L. T. (2004). Academic resilience in mathematics among poor and minority students. The Elementary School Journal, 177-195. doi: 10.1086/499748

Bråten, I., \& Strømsø, H. I. (2005). The relationship between epistemological beliefs, implicit theories of intelligence, and self-regulated learning among Norwegian postsecondary students. British Journal of Educational Psychology, 75(4), 539-565. doi: 10.1348/000709905X25067

Burnette, J. L., O’Boyle, E. H., Van Epps, E. M., Pollack, J. M., \& Finkel, E. J. (2013). Mind-sets matter: A meta-analytic review of implicit theories and self-regulation. Psychological Bulletin, 139(3), 655-701. doi: 10.1037/a0029531

Burns, K. C., \& Isbell, L. M. (2007). Promoting malleability is not one size fits all: Priming implicit theories of intelligence as a function of self-theories. Self and Identity, 6(1), 51-63. doi: $10.1080 / 15298860600823864$

Covington, M. V. (2000). Goal theory, motivation, and school achievement: An integrative review. Annual Review of Psychology, 51(1), 171-200.doi: 10.1146/annurev.psych.51.1.171

Crosnoe, R., Morrison, F., Burchinal, M., Pianta, R., Keating, D., Friedman, S. L., \& ClarkeStewart, K. A. (2010). Instruction, teacher-student relations, and math achievement trajectories in elementary school. Journal of Educational Psychology, 102(2), 407. doi: 10.1037/ a0017762

Cury, F., Da Fonseca, D., Zahn, I., \& Elliot, A. (2008). Implicit theories and IQ test performance: A sequential mediational analysis. Journal of Experimental Social Psychology, 44(3), 783791. doi: 10.1016/j.jesp.2007.07.003

Dupeyrat, C., \& Mariné, C. (2005). Implicit theories of intelligence, goal orientation, cognitive engagement, and achievement: A test of Dweck's model with returning to school adults. Contemporary Educational Psychology, 30(1), 43-59. doi: 10.1016/j.cedpsych.2004.01.007

Dweck, C. S. (1999). Self-theories: Their role in motivation, personality, and development. New York: Psychology Press.

Dweck, C. S., Chiu, C. Y., \& Hong, Y. Y. (1995). Implicit theories and their role in judgments and reactions: A word from two perspectives. Psychological Inquiry, 6(4), 267-285. doi: 10.1207/ s15327965pli0604_1

El-Alayli, A., \& Baumgardner, A. N. N. (2003). If at first you don't succeed, what makes you try, try again? Effects of implicit theories and ability feedback in a performance-oriented climate. Self and Identity, 2(2), 119-135. doi: 10.1080/15298860309031

Ferry, T. R., Fouad, N. A., \& Smith, P. L. (2000). The role of family context in a social cognitive model for career-related choice behavior: A math and science perspective. Journal of Vocational Behavior, 57(3), 348-364. doi: 10.1006/jvbe.1999.1743 
Fuller-Rowell, T. E., Evans, G. W., Paul, E., \& Curtis, D. S. (2015). The Role of Poverty and Chaos in the Development of Task Persistence Among Adolescents. Journal of Research on Adolescence, 25(4), 606-613. doi: 10.1111/jora.12157

Fulmer, S. M., \& Tulis, M. (2013). Changes in interest and affect during a difficult reading task: Relationships with perceived difficulty and reading fluency. Learning and Instruction, 27, 11-20. doi: 10.1016/j.learninstruc.2013.02.001

Ganley, C. M., \& Vasilyeva, M. (2014). The role of anxiety and working memory in

gender differences in mathematics. Journal of Educational Psychology, 106 (1), 105-120. doi: $10.1037 / \mathrm{a} 0034099$

Hackett, G. (1985). Role of mathematics self-efficacy in the choice of math-related majors of college women and men: A path analysis. Journal of Counseling Psychology, 32(1), 47. doi: 10.1037/0022-0167.32.1.47

Hardre, P. L., \& Reeve, J. (2003). A motivational model of rural students' intentions to persist in, versus drop out of, high school. Journal of Educational Psychology, 95(2), 347. doi: 10.1037/0022-0663.95.2.347

Heine, S. J., Kitayama, S., Lehman, D. R., Takata, T., Ide, E., Leung, C., \& Matsumoto, H. (2001). Divergent consequences of success and failure in Japan and North America: an investigation of self-improving motivations and malleable selves. Journal of Personality and Social Psychology, 81(4), 599-615. doi: 10.1037/0022-3514.81.4.599

Hong, Y. Y., Chiu, C. Y., Dweck, C. S., Lin, D. M. S., \& Wan, W. (1999). Implicit theories, attributions, and coping: A meaning system approach. Journal of Personality and Social Psychology, 77(3), 588-599. doi: 10.1037/0022-3514.77.3.588

Lee, J. (2002). Racial and ethnic achievement gap trends: Reversing the progress toward equity?. Educational Researcher, 31(1), 3-12. doi: 10.3102/0013189X031001003

Levy, S. R., Stroessner, S. J., \& Dweck, C. S. (1998). Stereotype formation and endorsement: The role of implicit theories. Journal of Personality and Social Psychology, 74(6), 1421. doi: 10.1037/0022-3514.74.6.1421

Liu, C. H., Chiu, F. C., Chen, H. C., \& Lin, C. Y. (2014). Helpful but insufficient: Incremental theory on challenge-confronting tendencies for students who fear being laughed at. Motivation and Emotion, 38(3), 367-377. doi: 10.1007/s11031-013-9386-x

Lucas, B. J., \& Nordgren, L. F. (2015). People underestimate the value of persistence for creative performance. Journal of Personality and Social Psychology, 109(2), 232-243. doi: 10.1037/ pspa0000030

Luzzo, D. A., Hasper, P., Albert, K. A., Bibby, M. A., \& Martinelli, Jr., E. A. (1999). Effects of self-efficacy-enhancing interventions on the math/science self-efficacy and career interests, goals, and actions of career undecided college students. Journal of Counseling Psychology, 46(2), 233. doi: 10.1037/0022-0167.46.2.233

Mazzocco, M. M., \& Kover, S. T. (2007). A longitudinal assessment of executive function skills and their association with math performance. Child Neuropsychology, 13(1), 18-45. doi: $10.1080 / 09297040600611346$

McClelland, M. M., Cameron, C. E., Connor, C. M., Farris, C. L., Jewkes, A. M., \& Morrison, F. J. (2007). Links between behavioral regulation and preschoolers' literacy, vocabulary, and math skills. Developmental Psychology, 43(4), 947. doi: 10.1037/0012-1649.43.4.947

Meece, J. L., Anderman, E. M., \& Anderman, L. H. (2006). Classroom goal structure, student motivation, and academic achievement. Annual Review of Psychology, 57, 487. doi: 10.1146/ annurev.psych.56.091103.070258

Miele, D. B., Son, L. K., \& Metcalfe, J. (2013). Children's naive theories of intelligence influence their metacognitive judgments. Child Development, 84(6), 1879-1886. doi: 10.1111/ cdev.12101 
Nussbaum, A. D., \& Steele, C. M. (2007). Situational disengagement and persistence in the face of adversity. Journal of Experimental Social Psychology, 43(1), 127-134. doi: 10.1016/j. jesp.2005.12.007

O'Brien, L. T., \& Crandall, C. S. (2003). Stereotype threat and arousal: Effects on women's math performance. Personality and Social Psychology Bulletin, 29(6), 782-789. doi: $10.1177 / 0146167203029006010$

O'Shea, A., Cleary, J., \& Breen, S. (2010). Exploring the role of confidence, theory of intelligence and goal orientation in determining a student's persistence on mathematical tasks in Joubert, M. and Andrews, P. (Eds.) Proceedings of the British Congress for Mathematics Education.

Reardon, S. F. (2011). The widening academic achievement gap between the rich and the poor: New evidence and possible explanations. Whither Opportunity, 91-116.

Shapka, J. D., Domene, J. F., \& Keating, D. P. (2006). Trajectories of career aspirations through adolescence and young adulthood: Early math achievement as a critical filter. Educational Research and Evaluation, 12(4), 347-358. doi: 10.1080/13803610600765752

Singh, K., Granville, M., \& Dika, S. (2002). Mathematics and science achievement: Effects of motivation, interest, and academic engagement. The Journal of Educational Research, 95(6), 323-332. doi: 10.1080/00220670209596607

Steinmayr, R., \& Spinath, B. (2009). The importance of motivation as a predictor of school achievement. Learning and Individual Differences, 19(1), 80-90. doi: 10.1016/j.lindif.2008.05.004

Swanson, H. L., \& Beebe-Frankenberger, M. (2004). The relationship between working memory and mathematical problem solving in children at risk and not at risk for serious math difficulties. Journal of Educational Psychology, 96(3), 471. doi: 10.1037/0022-0663.96.3.471

Thomas, M. S. C. (2009, June 10). A note on repeated measures ANCOVA. Retrieved from: http://www.psyc.bbk.ac.uk/research/DNL/stats/Repeated_Measures_ANCOVA.html

Thomas, M. S. C., Annaz, D., Ansari, D., Serif, G., Jarrold, C., \& Karmiloff-Smith, A. (2009). Using developmental trajectories to understand developmental disorders. Journal of Speech, Language, and Hearing Research, 52, 336-358. doi: 10.1044/1092-4388(2009/07-0144)

Vasilyeva, M., Laski, E. V., \& Shen, C. (2015). Computational fluency and strategy choice predict individual and cross-national differences in complex arithmetic. Developmental Psychology, 51(10), 1489. doi: 10.1037/dev0000045

Vermeer, H. J., Boekaerts, M., \& Seegers, G. (2000). Motivational and gender differences: Sixthgrade students' mathematical problem-solving behavior. Journal of Educational Psychology, 92(2), 308-315. doi: 10.1037/0022-0663.92.2.308

Wigfield, A., \& Meece, J. L. (1988). Math anxiety in elementary and secondary school students. Journal of Educational Psychology, 80(2), 210. doi: 10.1037/0022-0663.80.2.210

Original manuscript received February 24, 2016

Revised manuscript accepted March 13, 2016

First published online September 30, 2016 


\section{Appendix}

\section{Math Persistence Task}

\section{Solvable problem}

A car travels downhill at $72 \mathrm{mph}$ (miles per hour), on the level at $63 \mathrm{mph}$, and uphill at only $56 \mathrm{mph}$. The car takes 4 hours to travel from town A to town B. The return trip takes 4 hours and 40 minutes.

Find the distance between the two towns.

Solution: Let the total distance travelled downhill, on the level, and uphill, on the outbound journey, be $\mathrm{x}, \mathrm{y}$, and $\mathrm{z}$, respectively.

The time taken to travel a distance $\mathrm{s}$ at speed $\mathrm{v}$ is $\mathrm{s} / \mathrm{v}$.

Hence, for the outbound journey

$x / 72+y / 63+z / 56=4$

While for the return journey, which we assume to be along the same roads

$x / 56+y / 63+z / 72=14 / 3$

Multiplying both equations by the least common multiple of denominators 56,63 , and 72, we obtain

$$
\begin{aligned}
& 7 x+8 y+9 z=4 \cdot 7 \cdot 8 \cdot 9 \\
& 9 x+8 y+7 z=(14 / 3) \cdot 7 \cdot 8 \cdot 9
\end{aligned}
$$

Now it is clear that we should add the equations, yielding

$16(x+y+z)=(26 / 3) \cdot 7 \cdot 8 \cdot 9$

Therefore $x+y+z=273$; the distance between the two towns is 273 miles.

\section{Unsolvable problem}

Make 2 three-digit numbers using the following digits-1, 3, 4, 5, 6, 9-so that one of these three-digit numbers is exactly five times greater than the other (each digit should be used only once).

Enter your solution below and, on the next page, explain how you arrived at this solution.

Solution: Since one number has to be exactly five times greater than the other, the last digit of the bigger number has to be 0 or 5 . However, 0 is not on the available digit list, so it has to be 5 . Meanwhile, to make a three-digit number five times greater than the other, the hundreds place has to be $6,7,8$ or 9 . According to the given list, the possible bigger numbers would be $9 \times 5$ and $6 \times 5$.

At the same time, the hundreds place of the smaller number has to be 1, to keep the bigger number three-digit, and the ones place of the smaller number has to be an odd number, making the only possible smaller numbers $1 \times 3$.

Thus, there are four possible pairs: $965 \& 143,945 \& 163,645 \& 193$, and $695 \& 143$. None of those bigger three-digit numbers is exactly five times greater than the smaller one, meaning that there is no solution to this problem. 